\title{
Synovial sarcoma individual chemotherapy directed by collagen gel droplet embedded culture drug sensitivity test: A case report
}

\author{
FENG GU $^{1 *}$, YONGJIE MA ${ }^{2 *}$, YU FAN ${ }^{1}$, RONGGANG LANG ${ }^{1}$, TING DING $^{1}$, \\ XISHAN HAO ${ }^{1}$, YUN GONG ${ }^{3}$, HISAYUKI KOBAYAHSI ${ }^{4}$, ZHIWEI FANG $^{5}$ and LI FU $^{1}$ \\ ${ }^{1}$ Anti-tumor Drug Sensitivity Test Lab, Department of Breast Cancer Pathology and Research Laboratory, \\ Key Laboratory of Breast Cancer Prevention and Therapy of the Ministry of Education, Key Laboratory of Cancer \\ Prevention and Therapy of Tianjin; ${ }^{2}$ Central Laboratory of Oncology Department, Tianjin Medical University \\ Cancer Hospital, Tianjin 300060, P.R. China; ${ }^{3}$ Department of Pathology, University of Texas M. D. Anderson Cancer \\ Center, Houston, TX, USA; ${ }^{4}$ Nitta Gelatin Incorporation, Osaka, Japan; ${ }^{5}$ Department of Orthopedic Oncology, \\ Peking University School of Oncology, Beijing Cancer Hospital and Institute, Beijing 100142, P.R. China
}

Received April 15, 2010; Accepted June 18, 2010

DOI: 10.3892/ol_00000157

\begin{abstract}
The collagen gel droplet embedded culture-drug sensitivity test (CD-DST) is an anticancer drug sensitivity test developed about 10 years ago. This study reports the application of this test in the choice of neoadjuvant chemotherapy for the treatment of one patient with a large synovial sarcoma in the right shank. A 28-year old man presented at our hospital with a large mass in his right shank which had a hard texture and an obscure boundary. The histopathological diagnosis of excisional biopsy specimens was synovial sarcoma with low differentiation. Theprubicin/Cisplatin (THP/CDDP) neoadjuvant chemotherapy was selected based on the results obtained from the CD-DST. After three courses, a computed tomography (CT) scan was performed which indicated that the volume of the tumor had decreased significantly. Additionally, tumor necrosis, as well as the clinical response, showed complete response. The histopathological diagnosis of wide excision specimens indicated a grade III chemotherapy response. The patient was alive and without recurrence after a follow-up of 16 months. Our results indicated that the CD-DST is a useful tool for selecting neoadjuvant chemotherapeutic drugs for patients with synovial sarcoma.
\end{abstract}

Correspondence to: Dr Li Fu and Dr Zhiwei Fang, Department of Breast Pathology, Tianjin Medical University Cancer Institute and Hospital, Central Laboratory of Oncology Department, Tianjin Cancer Hospital, Huanhuxi Road, Tiyuanbei, Hexi, Tianjin 300060, P.R. China

E-mail: yongjiemagu@yahoo.com.cn.

${ }^{*}$ Contributed equally

Key words: synovial sarcoma, collagen gel droplet embedded culture-drug sensitivity test, neoadjuvant chemotherapy

\section{Introduction}

Synovial sarcoma is a type of malignant soft-tissue tumor that usually occurs in $80 \%$ of cases and affects knee and ankle joints in young adults. It also occurs in shoulder, elbow and hip regions. This neoplasm develops close to the joints, tendon sheaths and bursae, but it is rare for it to involve the synovial membrane. For the treatment of these tumors, the mainstay of curative therapy is the complete surgical resection of such tumor manifestations with negative histological margins. However, up to $50 \%$ of patients will develop distant metastases during the course of their disease (1). Regional radiotherapy or adjuvant chemotherapy following surgery is therefore crucial. In certain cases, surgery is difficult to perform due to the large volume of the tumor, and then neoadjuvant chemotherapy is considered. A screening method for the selection of effective anticancer drugs for individual patients may be useful, particularly for patients who have large tumors and need neoadjuvant chemotherapy to decrease the volume of the tumor for local excision. The collagen gel droplet embedded culutre-drug sensitivity test (CD-DST) is an anticancer drug sensitivity test that requires only a small number of cells $\left(3 \times 10^{3}\right.$ per droplet) (2-4). The clinical usefulness of CD-DST for colon (5), breast (6) and gastric (7) cancers and even certain rare cases of solid tumors, such as ovarian mature cystic teratoma with malignant transformation to adenocarcinoma (8), have been reported. Yabushita et al (9) found a strong correlation between clinical outcome and CD-DST results for anticancer drugs. This is a study of a case of a large synovial sarcoma in the right shank, for which neoadjuvant chemotherapy was investigated using CD-DST.

\section{Materials and methods}

Anticancer drugs. Isophosphamide (IFO) $(10,11)$ and anthracycline anti-tumor drugs (11,12), including therarubicin (THP), adriamycin (ADM) and epirubicin (EPI), are the first choice in the chemotherapy of soft tissue tumors. Cisplatin (CDDP) (13), vincristine (VCR) (13) and 5-fluorouracil (5-FU) 

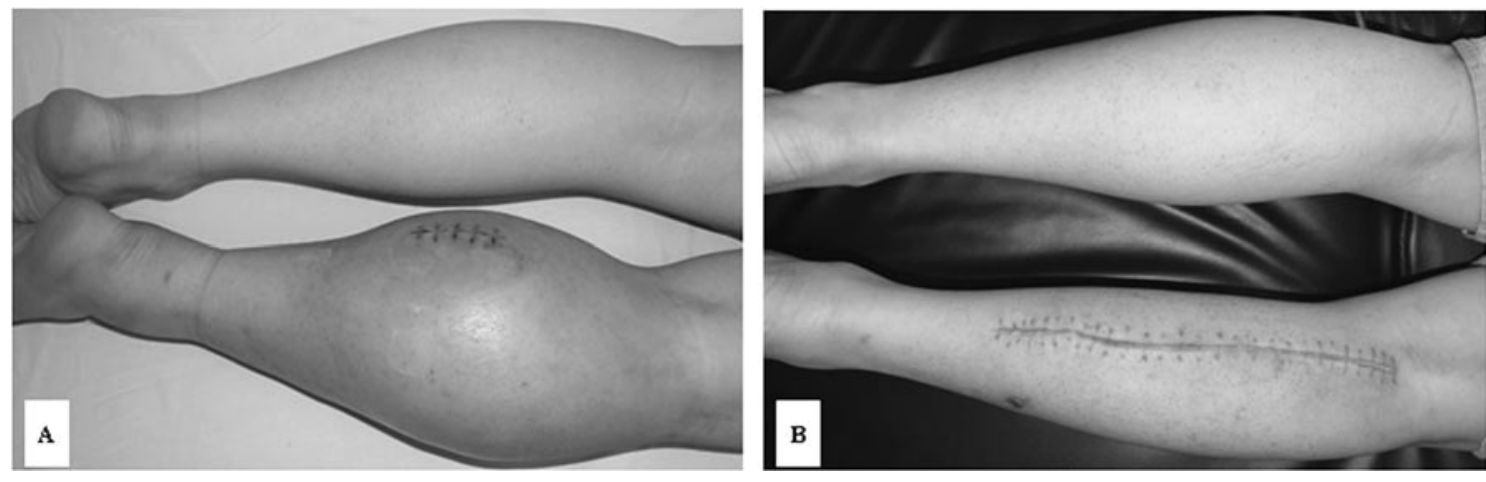

Figure 1. A large synovial sarcoma in the right shank. (A) Prior to and (B) after neoadjuvant chemotherapy and local tumor excision.
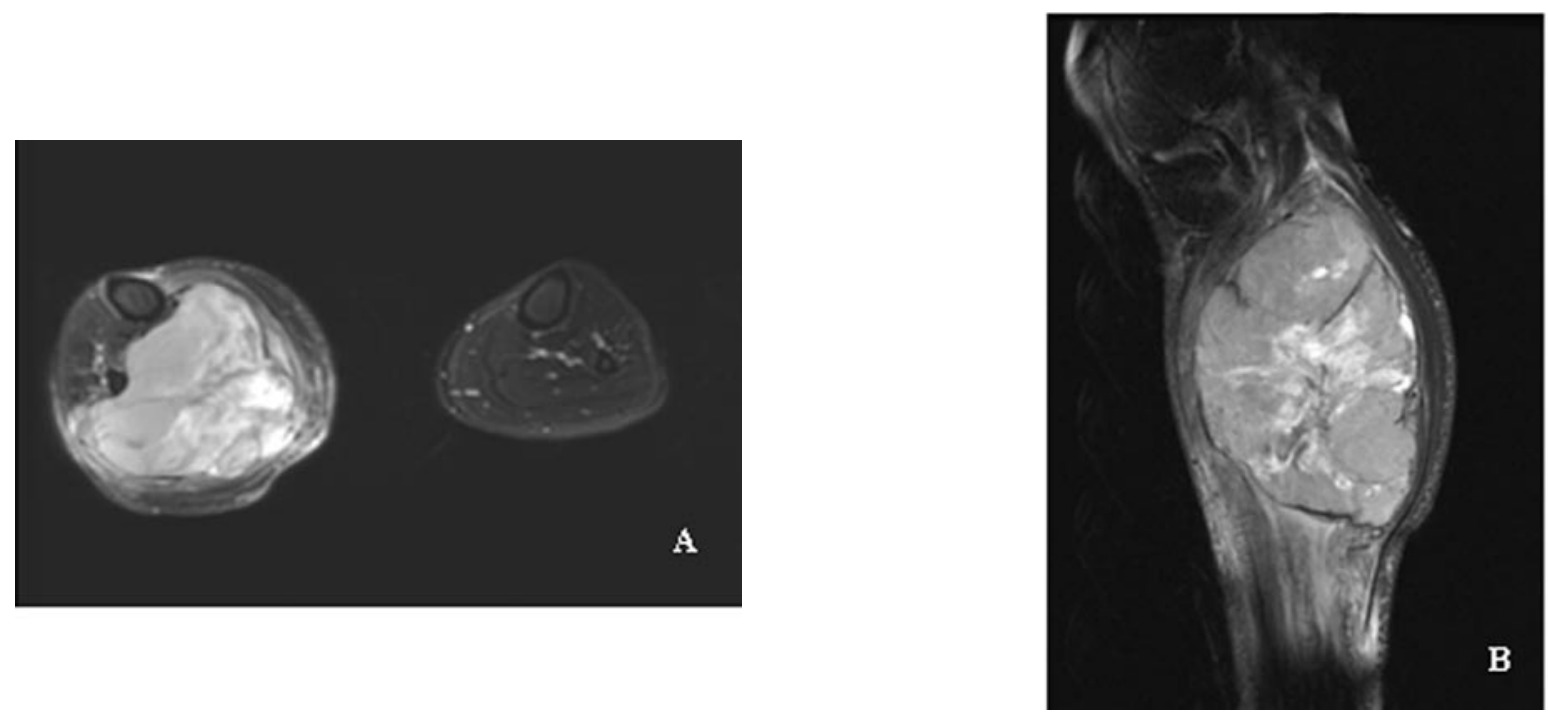

Figure 2. MRI of synovial sarcoma in the right shank prior to neoadjuvant chemotherapy. The sarcoma is $15 \times 10 \times 9 \mathrm{~cm}$, with an obscure boundary and uneven texture and without any destruction of the bone. (A) Axial plane. (B) Sagital plane.

$(14,15)$ have also been reported to be effective against soft tissue tumors. CDDP, THP, VCR and 5-FU were examined in CD-DST. IFO has no effect in in vitro tests, and since it was necessary for IFO to be activated by phosphamidase in vivo, it was used as a negative control.

Preparation of the tumor cell suspension. The excisional specimen was minced finely using scissors, suspended in Hank's balanced saline solution (HBSS, Gibco), treated with Dispersion Enzyme Cocktail EZ (including 1.0\% collagenase; Nitta Gelatin, Inc., Osaka, Japan) and digested at $37^{\circ} \mathrm{C}$ for $1 \mathrm{~h}$. The dispersed tumor cells were collected by centrifugation at $1000 \mathrm{rpm}$ for $3 \mathrm{~min}$, filtered through a $308 \mathrm{~nm}$ nylon mesh, washed in HBSS, suspended in PCM-1 medium (Nitta Gelatin) and then incubated in a collagen gel-coated flask (CG-flask, Nitta Gelatin) in a $\mathrm{CO}_{2}$ incubator at $37^{\circ} \mathrm{C}$ for $24 \mathrm{~h}$. The collagen gel in the CG-flask was dissolved in a cell dispersion using EZ, and only viable cells that adhered to the collagen gel were collected and used for the sensitivity test.

Collagen gel droplet embedded culture-drug sensitivity test. Type I collagen, 10X F-12 medium and reconstitution buffer (Cellmatrix Type CD, Nitta Gelatin) were combined in an ice bath at a ratio of 8:1:1. The prepared tumor cell suspension was added to a collagen solution $(1: 10, \mathrm{v}: \mathrm{v})$ at a final density of $2 \times 10^{5}$ cells $/ \mathrm{ml}$. A total of three drops of the collagen-cell mixture $(30 \mu \mathrm{l} / \mathrm{drop})$ were placed in each well of a 6-well multiplate on ice and allowed to gel at $37^{\circ} \mathrm{C}$ in a $\mathrm{CO}_{2}$ incubator; the final concentration was $\sim 3 \times 10^{3}$ cells/droplet. One hour later, each well was overlaid with $3 \mathrm{ml}$ DMEM/F 12 medium (Gibco) containing 10\% fetal bovine serum (Gibco, Canada), incubated in a $\mathrm{CO}_{2}$ incubator at $37^{\circ} \mathrm{C}$ overnight. CDDP, THP, VCR and 5-FU were then added to the final concentrations of 0.2 and $2.0 \mu \mathrm{g} / \mathrm{ml}, 0.02$ and $0.2 \mu \mathrm{g} / \mathrm{ml}, 0.01$ and $0.1 \mu \mathrm{g} / \mathrm{ml}$ and 1.0 and $10 \mu \mathrm{g} / \mathrm{ml}$, respectively, followed by further incubation for $24 \mathrm{~h}$. IFO (1 or $10 \mu \mathrm{g} / \mathrm{ml})$ was added as a negative control.

In vitro chemosensitivity test. After removal of the medium containing the anticancer drugs, each well was rinsed twice with $3 \mathrm{ml}$ HBSS, overlaid with $4 \mathrm{ml}$ PCM-2 medium (Serum Free Medium, Nitta Gelatin) and incubated for an additional 7 days. On the fourth day of incubation, the medium was changed. At the end of the incubation, neutral red (Nitta Gelatin) was added to each well at a final concentration of $50 \mu \mathrm{g} / \mathrm{ml}$, and cells in the collagen gel droplets were stained for $2 \mathrm{~h}$. Each collagen droplet was fixed with $10 \%$ neutral formalin buffer, 

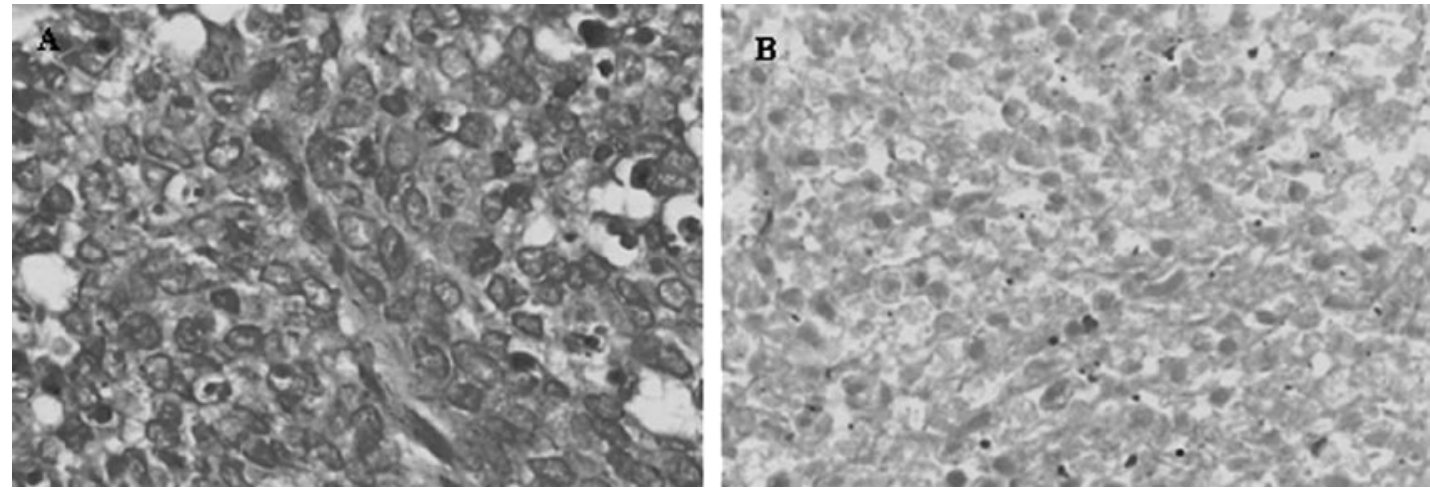

Figure 3. (A) H\&E staining of excisional biopsy specimens prior to neoadjuvant chemotherapy (magnification, x200). (B) H\&E staining of local tumor excision specimens after neoadjuvant chemotherapy. Fibroblast cell proliferation and tumor cell necrosis, grade III chemotherapy response (magnification, x200).

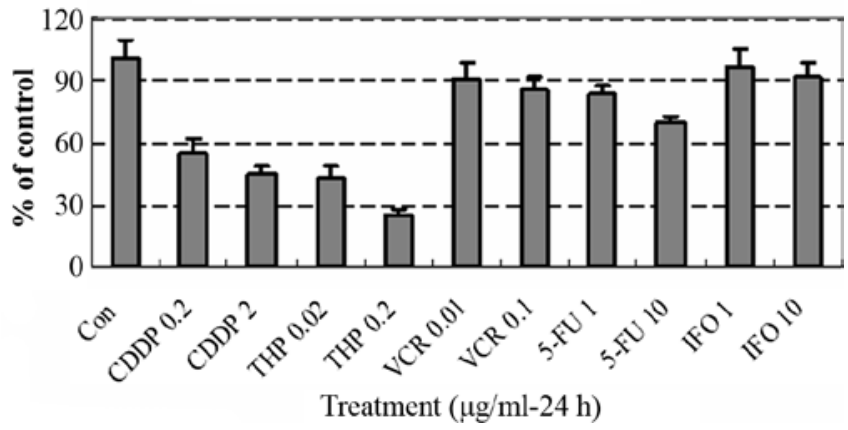

Figure 4. The survival rate of tumor cells and results of the CD-DST. CDDP and THP have a significant effect on the growth of tumor cells, while VCR and 5-FU show no significant effects. IFO was used as a negative control. Based on these results, CDDP/THP neoadjuvant chemotherapy was selected.

washed in double-distilled water, air-dried and quantified using image analysis. The in vitro sensitivity was expressed as the percentage of the $\mathrm{T} / \mathrm{C}$ ratio, where $\mathrm{T}$ is the total volume in the treated group and $\mathrm{C}$ is the total volume in the control group. When the $\mathrm{T} / \mathrm{C}$ ratio was $\leq 50 \%$, the in vitro drug sensitivity was regarded as effective. A $\mathrm{T} / \mathrm{C}$ ratio of $>50$ and $\leq 60 \%$ was considered borderline, and a $\mathrm{T} / \mathrm{C}$ ratio of $>60 \%$ was considered to indicate a lack of efficacy (2). Clinical responses were assessed according to World Health Organization criteria, whereby tumors demonstrating a complete response or partial response are considered clinically responsive. The results of the CD-DST are shown in Fig. 4.

\section{Results}

The patient was a 28-year-old male who presented with a large mass in the right shank. The perimeter of the right shank was $46.5 \mathrm{~cm}$, while the left side was $37 \mathrm{~cm}$ (Fig. 1A). Magnetic resonance imaging (MRI) revealed a $15 \times 10 \times 9 \mathrm{~cm}$ mass in the right shank (Fig. 2A and B), and a B-type Ultrasound scan showed a $1.0 \times 0.7 \times 0.5 \mathrm{~cm}$ intumesce in the right inguinal fold. The histopathological diagnosis of excisional biopsy specimens was synovial sarcoma with low differentiation (Fig. 3A).

Although the tumor was extremely large for local excision, the patient refused to accept amputation of the right extremity. Neoadjuvant chemotherapy was considered in order to decrease the volume of the tumor to allow for a local excision. A sample of $0.5 \mathrm{~g}$ of the excisional specimen was used for the CD-DST test.

Neoadjuvant THP/CDDP chemotherapy was selected based on the results of the CD-DST (Fig. 4), and administration of THP $\left(30 \mathrm{mg} / \mathrm{m}^{2}, \mathrm{~d} 1-\mathrm{d} 2\right)$ and CDDP $\left(40 \mathrm{mg} / \mathrm{m}^{2}\right.$, d1-d2) was initiated. Upon completion of three courses of THP/CDDP chemotherapy, the perimeter of the right shank decreased from 46.5 to $42.5 \mathrm{~cm}$, and its texture became much more supple. A precontrast CT scan showed that the volume of the tumor had decreased to $11 \times 8.2 \mathrm{~cm}$, while a contrast scan indicated tumor necrosis (Fig. 5A and B) and
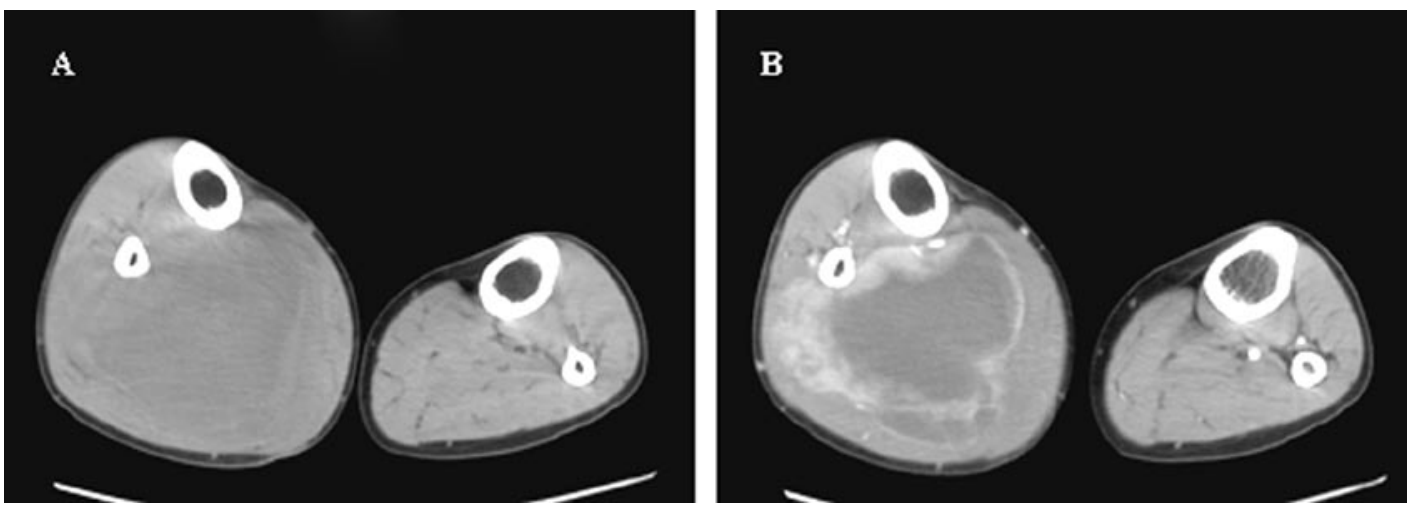

Figure 5. A CT scan of a synovial sarcoma in the right shank after neoadjuvant chemotherapy. (A) A precontrast scan showed that the volume of the sarcoma had decreased to $11 \times 8.2 \mathrm{~cm}$, and (B) a contrast scan revealed tumor necrosis. 
a B-type Ultrasound scan showed that the intumesce in the right inguinal fold had disappeared. Since the neoadjuvant THP/CDDP chemotherapy was successful, a local tumor excision was performed (Fig. 1B). The pathodiagnosis indicated fibroblast cell proliferation and tumor cell necrosis as well as a grade III chemotherapy response (Fig. 3B). Post-operatively, the patient received further adjuvant chemotherapy of THP/ CDDP, and he was alive and without recurrence at a 16-month follow-up.

\section{Discussion}

The first choice for the treatment of synovial sarcoma is local excision with wide margins of normal tissue, followed by regional radiotherapy or adjuvant chemotherapy $(16,17)$. However, in some cases surgery is difficult to perform due to the large volume of the tumor, thus neoadjuvant chemotherapy is considered. In the case we reported, the tumor was extremely large and we were unable to perform a local excision. The patient involved was young and refused to accept amputation of the extremity. Neoadjuvant chemotherapy was therefore considered in order to decrease the volume of the tumor. IFO $(10,11)$ and anthracycline anti-tumor drugs $(11,12)$, including THP, ADM and EPI, are the first choice in the chemotherapy of soft tissue tumors. CDDP (13), VCR (13) and 5-FU $(14,15)$ are also reported to be effective against soft tissue tumors. In this case, we used the system of CD-DST described by Kobayashi et al (2-4) to check the sensitivity of tumor cells to THP, VCR, CDDP and 5-FU. Since IFO should be activated by phosphamidase in vivo, we used it as the negative control. Based on the results of the CD-DST, THP/CDDP neoadjuvant chemotherapy was administered. Upon completion of three courses of THP/CDDP chemotherapy, the perimeter of the right shank decreased from 46.5 to $42.5 \mathrm{~cm}$, the texture became much more supple, and a CT scan showed that the volume of the tumor had decreased from $15 \times 10$ to $11 \times 8.2 \mathrm{~cm}$ and that the tumor necrosis had disappeared. A B-type Ultrasound scan showed that the intumesce in the right inguinal fold had also disappeared. The pathodiagnosis of the local excision specimen indicated fibroblast cell proliferation and tumor cell necrosis, as well as a grade III chemotherapy response (Fig. 3B). The patient was alive and without recurrence at a 16-month follow-up evaluation. This clinical course therefore correlated successfully with the results of the CD-DST. Our results show that $\mathrm{CD}$-DST can be used to evaluate anticancer drug sensitivity. Further investigation of CD-DST is required as a test for the prediction of anticancer drug sensitivity in cancer patients. CD-DST may therefore be particularly useful for selecting chemotherapeutic drugs for patients with synovial sarcoma, particularly for those with large tumors who require neoadjuvant chemotherapy to decrease the volume of the tumor prior to local excision surgery.

\section{References}

1. Schmitt T and Kasper B: New medical treatment options and strategies to assess clinical outcome in soft-tissue sarcoma. Expert Rev Anticancer Ther 9: 1159-1167, 2009.
2. Kobayashi $\mathrm{H}$, Higashiyama M, Minamigawa $\mathrm{K}$, Tanisaka $\mathrm{K}$, Takano T, Yokouchi H, Kodama K and Hata T: Examination of in vitro chemosensitivity test using collagen gel droplet culture method with colorimetric endpoint quantification. Jpn J Cancer Res 92: 203-210, 2001.

3. Kobayashi H: Development of a new in vitro chemosensitivity test using collagen gel droplet embedded culture and image analysis for clinical usefulness. Recent Results Cancer Res 161: 48-61, 2003.

4. Kobayashi H: Collagen gel droplet culture method to examine in vitro chemosensitivity. Methods Mol Med 110: 59-67, 2005.

5. Araki Y, Isomoto H, Matsumoto A, Kaibara A, Yasunaga M, Hayashi K, Yatsugi H and Yamauchi K: An in vitro chemosensitivity test for colorectal cancer using collagen-gel droplet embedded cultures. Kurume Med J 46: 163-166, 1999.

6. Tamura Y, Kobayashi H, Taguchi T, Motoyama K, Inaji H and Noguchi S: Prediction of chemotherapeutic response by collagen gel droplet embedded culture-drug sensitivity test in human breast cancers. Int J Cancer 98: 450-455, 2002.

7. Hanatani Y, Kobayashi H, Kodaira S, Takami H, Asagoe T and Kaneshiro E: An in vitro chemosensitivity test for gastric cancer using collagen gel droplet embedded culture. Oncol Rep 7: 1027-1033, 2000.

8. Yamagami W, Banno K, Kawaguchi M, Yanokura M, Kuwabara Y, Hirao N, Susumu N, Tsukazaki K and Aoki D: Use of the collagen gel droplet embedded drug sensitivity test to determine drug sensitivity against ovarian mature cystic teratoma with malignant transformation to adenocarcinoma: a case report. Chemotherapy 53: 137-141, 2007.

9. Yabushita H, Ohnishi M, Komiyama M, Mori T, Noguchi M, Kishida T, Noguchi Y, Sawaguchi K and Noguchi M: Usefulness of collagen gel droplet embedded culture drug sensitivity testing in ovarian cancer. Oncol Rep 12: 307-311, 2004.

10. Macdermed DM, Miller LL, Peabody TD, Simon MA, Luu HH, Haydon RC, Montag AG, Undevia SD and Connell PP: Primary tumor necrosis predicts distant control in locally advanced soft-tissue sarcomas after preoperative concurrent chemoradiotherapy. Int J Radiat Oncol Biol Phys 15: 1147-1153, 2010.

11. Kopp HG, Patel S, Brücher B and Hartmann JT: Potential combination chemotherapy approaches for advanced adult-type soft-tissue sarcoma. Am J Clin Dermatol 9: 207-217, 2008.

12. Patel S, Keohan ML, Saif MW, Rushing D, Baez L, Feit K, DeJager R and Anderson S: Phase II study of intravenous TZT-1027 in patients with advanced or metastatic soft-tissue sarcomas with prior exposure to anthracycline-based chemotherapy. Cancer 107: 2881-2887, 2006.

13. Goto T, Kosaku H, Kobayashi H, Hozumi T and Kondo T: Soft tissue sarcoma: postoperative chemotherapy. (In Japanese) Gan To Kagaku Ryoho 31: 1324-1330, 2004.

14. Wada Y, Hirayama Y, Seki R, Konuma Y, Kohda K, Yoshida M, Nakamura Y, Obata $M$ and Ando M: Long-term remission survival with a case of rectal carcinoid tumor with metastasis in the soft tissue effectively treated with the combination therapy of irinotecan/5-fluorouracil/levofolinate followed by resection. (In Japanese) Nippon Naika Gakkai Zasshi 96: 2513-2515, 2007.

15. Marchal JA, Boulaiz H, Rodríguez-Serrano F, Peran M, Carrillo E, Vélez C, Domínguez J, Gómez-Vidal JA, Campos J, Gallo MA, Espinosa A and Aránega A: 5-Fluorouracil derivatives induce differentiation mediated by tubulin and HLA class I modulation. Med Chem 3: 233-239, 2007.

16. Kopp HG, Patel S, Brucher B, Hartmann JT: Potential combination chemotherapy approaches for advanced adult-type soft-tissue sarcoma. Am J Clin Dermatol 9: 207-217, 2008.

17. Pervaiz N, Colterjohn N, Farrokhyar F, Tozer R, Figueredo A and Ghert M: A systematic meta-analysis of randomized controlled trails of adjuvant chemotherapy for localized resectable softtissue sarcoma. Cancer 113: 573-581, 2008. 